\title{
Secreted miR-210-3p as non-invasive biomarker in clear cell renal cell carcinoma
}

\author{
Vincenzo Petrozza ${ }^{1, *}$, Antonio Luigi Pastore ${ }^{2, *}$, Giovanni Palleschi $^{2}$, Claudia Tito ${ }^{3}$, \\ Natale Porta ${ }^{1}$, Serena Ricci ${ }^{4}$, Chiara Marigliano ${ }^{5}$, Manuela Costantini ${ }^{6,7}$, Giuseppe \\ Simone ${ }^{7}$, Angelina Di Carlo ${ }^{4}$, Michele Gallucci' ${ }^{7}$ Antonio Carbone ${ }^{2, *}$ and Francesco \\ $\mathbf{F a z i}^{3, *}$ \\ ${ }^{1}$ Department of Medico-Surgical Sciences and Biotechnologies, Sapienza University of Rome, Pathology Unit ICOT, Latina, \\ Italy \\ ${ }^{2}$ Department of Medico Surgical Sciences and Biotechnologies, Sapienza University of Rome, Urology Unit ICOT, Latina, Italy \\ ${ }^{3}$ Department of Anatomical, Histological, Forensic \& Orthopaedic Sciences, Section of Histology \& Medical Embryology, \\ Sapienza University of Rome, Rome, Italy \\ ${ }^{4}$ Department of Medico-Surgical Sciences and Biotechnologies, Sapienza University of Rome, Latina, Italy \\ ${ }^{5}$ Department of Radiological Sciences, Oncology and Pathology, Azienda Policlinico Umberto I, Sapienza University of Rome, \\ Rome, Italy \\ ${ }^{6}$ Department of Biosciences, Biotechnologies and Biopharmaceutics, University of Bari, Bari, Italy \\ ${ }^{7}$ Department of Urology, Regina Elena National Cancer Institute of Rome, Rome, Italy \\ *These authors have contributed equally to this work \\ Correspondence to: Francesco Fazi, email: francesco.fazi@uniroma l.it \\ Antonio Carbone, email: antonio.carbone@uniromal.it \\ Keywords: microRNAs, biomarkers, cCRCC, miR-210-3p, urine specimens
}

Received: January 16, $2017 \quad$ Accepted: May 06, $2017 \quad$ Published: June 13, 2017

Copyright: Petrozza et al. This is an open-access article distributed under the terms of the Creative Commons Attribution License 3.0 (CC BY 3.0), which permits unrestricted use, distribution, and reproduction in any medium, provided the original author and source are credited.

\section{ABSTRACT}

The most common subtype of renal cell carcinoma (RCC) is clear cell RCC (CCRCC). It accounts for $\mathbf{7 0 - 8 0 \%}$ of all renal malignancies representing the third most common urological cancer after prostate and bladder cancer. The identification of non-invasive biomarkers for the diagnosis and responsiveness to therapy of CCRCC may represent a relevant step-forward in CCRCC management. The aim of this study is to evaluate whether specific miRNAs deregulated in CCRCC tissues present altered levels also in urine specimens. To this end we first assessed that miR-21-5p, miR-210-3p and miR-221-3p resulted upregulated in ccRCC fresh frozen tissues compared to matched normal counterparts. Next, we evidenced that miR-210-3p resulted significantly upregulated in $\mathbf{3 8}$ urine specimens collected from two independent cohorts of ccRCC patients at the time of surgery compared to healthy donors samples. Of note, miR210-3p levels resulted significantly reduced in follow-up samples. These results point to miR-210-3p as a potential non-invasive biomarker useful not only for diagnosis but also for the assessment of complete resection or response to treatment in ccRCC management.

\section{INTRODUCTION}

Renal cell carcinoma (RCC) represents $2-3 \%$ of adult malignancies [1], with a peak of incidence occurring between 60 and 70 years and a 3:2 male:female ratio [2]. The clear cell RCC (ccRCC) is the most common subtype of RCC and represents the third most common urological cancer after prostate and bladder cancer. The incidence of the disease has been steadily rising in Europe to over 30,000 new cases per year and its mortality rate has reached $40 \%$ [3]. The early diagnosis and complete surgical excision are the main factors contributing to a 
definitive treatment of ccRCC. Surgery represents the most important therapeutic option for ccRCC management [4], but nephrectomy of the primary tumor is curative only if the tumor is still localized in the kidney. Thus, for patients with advanced and metastatic ccRCC, surgery is often palliative [5]. Targeted therapy has recently emerged in ccRCC management considering also that ccRCC is usually resistant to both chemotherapy and radiotherapy. Tyrosine kinases or mTOR pathways inhibitors are drugs often used in advanced RCC [6, 7]; however, resistance frequently occurs after these targeted therapies [8]. A key challenge for the improvement of ccRCC management could derive from a deeper molecular characterization of this neoplasm that could greatly improve the diagnosis, prognosis and treatment choice [9]. The comprehension of microRNAs (miRNAs) contribution to tumorigenesis and the identification of miRNAs as potential biomarkers for diagnosis and therapy response are becoming relevant for cancer management $[10,11]$. To date, a number of studies identified miRNAs differentially expressed between normal and neoplastic tissues in ccRCC as well as miRNAs secreted into blood and urine, suggesting that these small molecules may serve as non-invasive diagnostic, prognostic and surveillance markers in urological carcinomas [12-17]. The urinary miR-210 is currently under investigation as a potential tool for ccRCC management [18].

Recently, by using a retrospective cohort of 20 formalin-fixed paraffin-embedded (FFPE) tissues, we evaluated the levels of specific miRNAs (miR21-5p, miR-210-3p, miR185-5p and miR-221-3p) differentially expressed in ccRCC $v s$ matched normal tissues. We evidenced miR-21-5p and miR-210-3p as the most significantly up-regulated in this patient cohort, highlighting these onco-miRNAs as possible relevant players involved in ccRCC tumorigenesis [19]. To further support the potential clinical usefulness of these miRNAs in ccRCC management we started a prospective study. By using two independent cohorts of patients we evidenced that, among the miRNAs previously identified as up-regulated in ccRCC tissues, only miR-210-3p resulted significantly up-regulated in urine specimens collected from $38 \mathrm{ccRCC}$ patients at the time of surgery compared to healthy donors samples. Of note, miR210-3p levels resulted significantly reduced in followup samples, highlighting this onco-miRNA as potential biomarker useful not only for diagnosis but also to assess complete resection or response to treatment in ccRCC management.

\section{RESULTS}

By using a cohort of fresh frozen tissues obtained from $10 \mathrm{ccRCC}$ patients undergoing surgical nephrectomy resection we evaluated the expression of the same miRNAs analyzed in our previous retrospective study
[19]. The characteristics of ccRCC patients and tumor specimens are reported in the Methods section and summarized in Table 1. In agreement with the previous retrospective study we evidenced that miR-21-5p, miR-210-3p and miR-221-3p resulted up-regulated in these ccRCC vs matched adjacent normal fresh frozen tissues, while miR-185-5p and miR-145-5p did not show significant modulation (Figure 1A and Supplementary Figure 1A). Our data evidenced that miR-210-3p resulted the most significantly up-regulated miRNA in this patients cohort $(p$-value $=0.0149)($ Figure 1A).

To investigate whether the miRNAs deregulated in ccRCC tissues may serve as useful clinical biomarkers, we selected miR-21-5p, miR-210-3p and miR-221$3 p$ to evaluate their expression in 38 urine specimens collected at the time of surgery and during follow-up (with 15 months as median of time from surgery) from two independent cohorts of ccRCC patients; urine specimens from healthy donors were analyzed as well.

Of note, miR-210-3p resulted significantly upregulated in urine specimens collected from ccRCC patients at the time of surgery, compared to healthy donors samples (Figure 1B), contrarily to miR-21-5p and miR-221-3p, which didn't show increased levels in urine despite their trend of up-regulation observed in tumor tissues.

Importantly, miR-210-3p levels resulted significantly reduced during follow-up in six ccRCC patients of the same cohort, for which follow-up urine samples were available (Figure 1C). miR-21-5p and miR-221-3p resulted unchanged also in the follow-up samples (Figure 1C).

\section{DISCUSSION}

The identification of molecular markers in body fluids (e.g., sera and urine), which can be used as noninvasive diagnostic, prognostic and surveillance markers in ccRCC management, is one of the most ambitious challenges in oncologic research [20]. Of note, also miRNAs are acquiring a great potential as novel cancer biomarkers in urological carcinomas [16, 21-25]. To date, the increased interest on non-invasive biomarkers, allowed by use of novel methodologies (such as next generation sequencing, single-cell sequencing approaches and digital PCR) has greatly improved the translational potential of miRNAs into clinical research [16]. However, the development of a standardized method considering different methodological drawbacks (including samples collection, processing and storage or RNA isolation, quantification and normalization) is strongly needed to support the strength of circulating miRNAs as useful biomarkers in ccRCC management $[16,25]$.

In this study we show that miR-210-3p that was previously evidenced as up-regulated in ccRCC $v s$ matched normal tissues [19] also show increased levels 
Table 1: Clinical characteristics of patients with ccRCC

\begin{tabular}{|c|c|c|c|c|c|c|c|c|c|c|}
\hline $\begin{array}{l}\text { Gender/ } \\
\text { age }\end{array}$ & $\begin{array}{l}\text { Weight/ } \\
\text { height } \\
(\mathrm{kg} / \mathrm{cm})\end{array}$ & $\begin{array}{l}\text { Smoking } \\
\text { history }\end{array}$ & $\begin{array}{l}\text { Serum } \\
\text { creatinine } \\
(\mathbf{m g} / \mathbf{d l})\end{array}$ & Histology & $\begin{array}{c}\text { Tumor } \\
\text { grade } \\
\text { (Fuhrman) }\end{array}$ & $\begin{array}{l}\text { Pathologic } \\
\text { stage }\end{array}$ & $\begin{array}{c}\text { Tumor } \\
\text { size } \\
\text { (cm) }\end{array}$ & $\begin{array}{l}\text { L. Nodes } \\
\text { Inv. }\end{array}$ & Metast. & Surgery \\
\hline $\mathrm{M} / 52^{*}$ & $70 \mathrm{kgx} 165 \mathrm{~cm}$ & No & 1.11 & $\mathrm{ccRCC}$ & $\mathrm{G} 2$ & pT2 & 8.0 & $\mathrm{Nx}$ & Mx & $\begin{array}{l}\text { Lap. Rad. Right } \\
\text { Neph. }\end{array}$ \\
\hline $\mathrm{F} / 73 * *$ & $65 \mathrm{kgx} 160 \mathrm{~cm}$ & No & 0.88 & $\mathrm{ccRCC}$ & $\mathrm{G} 2$ & pT1a & 2.0 & $\mathrm{Nx}$ & Mx & $\begin{array}{c}\text { Lap. Rad. Right } \\
\text { Neph. }\end{array}$ \\
\hline $\mathrm{M} / 65^{*}$ & $75 \mathrm{~kg} \times 170 \mathrm{~cm}$ & No & 1.00 & $\mathrm{ccRCC}$ & $\mathrm{G} 2$ & pT1a & 2.0 & $\mathrm{Nx}$ & $\mathrm{Mx}$ & $\begin{array}{l}\text { Lap. Part. Right } \\
\text { Neph. }\end{array}$ \\
\hline $\mathrm{M} / 74^{* *}$ & $75 \mathrm{~kg} \times 165 \mathrm{~cm}$ & No & 0.84 & $\mathrm{ccRCC}$ & $\mathrm{G} 2$ & pT1a & nd & $\mathrm{Nx}$ & $\mathrm{Mx}$ & $\begin{array}{l}\text { Lap. Part. Left } \\
\text { Neph. }\end{array}$ \\
\hline $\mathrm{M} / 84^{* *}$ & $90 \mathrm{~kg} \times 165 \mathrm{~cm}$ & No & 0.75 & $\mathrm{ccRCC}$ & $\mathrm{G} 2$ & pT1a & 3.0 & $\mathrm{Nx}$ & Mx & $\begin{array}{l}\text { Lap. Rad. Right } \\
\text { Neph. }\end{array}$ \\
\hline $\mathrm{M} / 70^{*}$ & $85 \mathrm{~kg} \times 170 \mathrm{~cm}$ & Yes & 0.99 & $\mathrm{ccRCC}$ & $\mathrm{G} 2$ & pT3a & 7.5 & $\mathrm{Nx}$ & Mx & $\begin{array}{l}\text { Lap. Rad. Left } \\
\text { Neph. }\end{array}$ \\
\hline $\mathrm{M} / 70^{* *}$ & $78 \mathrm{~kg} \times 175 \mathrm{~cm}$ & Yes & 1.55 & $\mathrm{ccRCC}$ & G2 & pT1a & 2.5 & $\mathrm{Nx}$ & Mx & $\begin{array}{l}\text { Lap. Part. Right } \\
\text { Neph. }\end{array}$ \\
\hline $\mathrm{M} / 42 * *$ & $60 \mathrm{~kg} \times 170 \mathrm{~cm}$ & Yes & 1.13 & $\mathrm{ccRCC}$ & $\mathrm{G} 2$ & pT3a & 9.5 & No & $\mathrm{Mx}$ & $\begin{array}{l}\text { Lap. Rad. Left } \\
\text { Neph. }\end{array}$ \\
\hline $\mathrm{F} / 36^{* *}$ & $50 \mathrm{~kg} \times 155 \mathrm{~cm}$ & No & 2.70 & $\mathrm{ccRCC}$ & $\mathrm{G} 2$ & pT1b & 5.0 & $\mathrm{Nx}$ & Mx & $\begin{array}{l}\text { Lap. Rad. Left } \\
\text { Neph. }\end{array}$ \\
\hline $\mathrm{M} / 81^{*}$ & $80 \mathrm{~kg} \mathrm{x} 174 \mathrm{~cm}$ & Yes & 1.01 & ccRCC & $\mathrm{G} 2$ & pT1b & 4.3 & $\mathrm{Nx}$ & $\mathrm{Mx}$ & $\begin{array}{l}\text { Lap. Rad. Left } \\
\text { Neph. }\end{array}$ \\
\hline $\mathrm{F} / 64^{\dagger}$ & $99 \mathrm{~kg} \times 180 \mathrm{~cm}$ & Yes & 1.3 & $\mathrm{ccRCC}$ & G3 & pT3a & 6 & No & $\mathrm{Mx}$ & $\begin{array}{c}\text { Lap. Rad. Right } \\
\text { Neph. }\end{array}$ \\
\hline $\mathrm{M} / 74^{\dagger}$ & $73 \mathrm{~kg} \times 160 \mathrm{~cm}$ & Former & 1.21 & $\mathrm{ccRCC}$ & G3 & pT1a & 3 & $\mathrm{Nx}$ & Mx & $\begin{array}{l}\text { Lap. Part. Right } \\
\text { Rob. Neph. }\end{array}$ \\
\hline $\mathrm{M} / 51^{\dagger}$ & $85 \mathrm{~kg} \times 185 \mathrm{~cm}$ & Former & 1.19 & ccRCC & G3 & pT2a & 8 & No & Mx & $\begin{array}{l}\text { Lap. Rad. Left } \\
\text { Neph. }\end{array}$ \\
\hline $\mathrm{M} / 61^{\dagger}$ & $92 \mathrm{~kg} \mathrm{x} 165 \mathrm{~cm}$ & Former & 1.32 & $\mathrm{ccRCC}$ & G3 & pT3b & 7 & $\mathrm{Nx}$ & M1 & $\begin{array}{l}\text { Lap. Rad. Right } \\
\text { Rob. Neph. }\end{array}$ \\
\hline $\mathrm{M} / 42^{\dagger}$ & $\mathrm{n} / \mathrm{a}$ & Yes & 1.36 & $\mathrm{ccRCC}$ & G3 & pT3a & 7.5 & $\mathrm{Nx}$ & $\mathrm{Mx}$ & $\begin{array}{l}\text { Lap. Rad. Left } \\
\text { Neph }\end{array}$ \\
\hline $\mathrm{M} / 74^{\dagger}$ & $84 \mathrm{~kg} \times 183 \mathrm{~cm}$ & No & 1.1 & ccRCC & G3 & pT3a & 5.4 & $\mathrm{Nx}$ & Mx & $\begin{array}{l}\text { Lap. Part. Left } \\
\text { Rob. Neph. }\end{array}$ \\
\hline $\mathrm{F} / 61^{\dagger}$ & $\mathrm{n} / \mathrm{a}$ & $\mathrm{n} / \mathrm{a}$ & 1.06 & $\mathrm{ccRCC}$ & G3 & pT3a & 5 & $\mathrm{Nx}$ & M1 & $\begin{array}{l}\text { Lap. Rad. Right. } \\
\text { Neph. }\end{array}$ \\
\hline $\mathrm{F} / 77^{\dagger}$ & $78 \mathrm{~kg} \times 165 \mathrm{~cm}$ & No & 1.1 & $\mathrm{ccRCC}$ & G4 & pT1b & 6 & $\mathrm{Nx}$ & Mx & $\begin{array}{l}\text { Lap. Rad. Left } \\
\text { Neph. }\end{array}$ \\
\hline $\mathrm{M} / 73^{\dagger}$ & $90 \mathrm{~kg} \times 175 \mathrm{~cm}$ & No & 1.4 & ccRCC & G3 & pT1a & 4 & $\mathrm{Nx}$ & $\mathrm{Mx}$ & $\begin{array}{l}\text { Lap. Part. Left } \\
\text { Rob. Neph. }\end{array}$ \\
\hline $\mathrm{M} / 62^{\dagger}$ & $69 \mathrm{~kg} \times 164 \mathrm{~cm}$ & No & 1.18 & $\mathrm{ccRCC}$ & $\mathrm{G} 2$ & $\mathrm{pT} 2 \mathrm{a}$ & 7.2 & $\mathrm{Nx}$ & Mx & $\begin{array}{l}\text { Lap. Part. Right } \\
\text { Rob. Neph. }\end{array}$ \\
\hline $\mathrm{M} / 45^{\dagger}$ & $69 \mathrm{~kg} \times 170 \mathrm{~cm}$ & Yes & 1 & $\mathrm{ccRCC}$ & G2 & pT1a & 2.5 & $\mathrm{Nx}$ & Mx & $\begin{array}{l}\text { Lap. Part. Right } \\
\text { Rob. Neph. }\end{array}$ \\
\hline
\end{tabular}




\begin{tabular}{|c|c|c|c|c|c|c|c|c|c|c|}
\hline $\begin{array}{l}\text { Gender/ } \\
\text { age }\end{array}$ & $\begin{array}{c}\text { Weight/ } \\
\text { height } \\
(\mathrm{kg} / \mathrm{cm})\end{array}$ & $\begin{array}{l}\text { Smoking } \\
\text { history }\end{array}$ & $\begin{array}{c}\text { Serum } \\
\text { creatinine } \\
(\mathrm{mg} / \mathrm{dl})\end{array}$ & Histology & $\begin{array}{c}\text { Tumor } \\
\text { grade } \\
\text { (Fuhrman) }\end{array}$ & $\begin{array}{l}\text { Pathologic } \\
\text { stage }\end{array}$ & $\begin{array}{c}\text { Tumor } \\
\text { size } \\
\text { (cm) }\end{array}$ & $\begin{array}{l}\text { L. Nodes } \\
\text { Inv. }\end{array}$ & Metast. & Surgery \\
\hline $\mathrm{F} / 62^{\dagger}$ & $80 \mathrm{~kg} \times 160 \mathrm{~cm}$ & No & 5.5 & $\mathrm{ccRCC}$ & G2 & pT1b & 0.88 & $\mathrm{Nx}$ & $\mathrm{Mx}$ & $\begin{array}{l}\text { Lap. Part. Right } \\
\text { Rob. Neph. }\end{array}$ \\
\hline $\mathrm{F} / 82^{\dagger}$ & $65 \mathrm{~kg} \times 155 \mathrm{~cm}$ & No & 0.66 & $\mathrm{ccRCC}$ & G2 & pT1a & 3.5 & $\mathrm{Nx}$ & $\mathrm{Mx}$ & $\begin{array}{l}\text { Lap. Part. Right } \\
\text { Rob. Neph. }\end{array}$ \\
\hline $\mathrm{M} / 53^{\dagger}$ & $71 \mathrm{~kg} \times 168 \mathrm{~cm}$ & Yes & 1.12 & $\mathrm{ccRCC}$ & G4 & pT1b & 6 & $\mathrm{Nx}$ & M1 & $\begin{array}{l}\text { Lap. Rad. Left } \\
\text { Neph. }\end{array}$ \\
\hline $\mathrm{F} / 68^{+}$ & $80 \mathrm{~kg} \times 160 \mathrm{~cm}$ & Former & 0.89 & $\mathrm{ccRCC}$ & G2 & pT1b & 5 & $\mathrm{Nx}$ & $\mathrm{Mx}$ & $\begin{array}{l}\text { Lap. Part. Left } \\
\text { Rob. Neph. }\end{array}$ \\
\hline $\mathrm{F} / 64^{\dagger}$ & $\mathrm{n} / \mathrm{a}$ & $\mathrm{n} / \mathrm{a}$ & 1 & $\mathrm{ccRCC}$ & G2 & pT1a & 3 & $\mathrm{Nx}$ & $\mathrm{Mx}$ & $\begin{array}{l}\text { Lap. Part. Left } \\
\text { Rob. Neph. }\end{array}$ \\
\hline $\mathrm{F} / 78^{\dagger}$ & $\mathrm{n} / \mathrm{a}$ & $\mathrm{n} / \mathrm{a}$ & 0.86 & $\mathrm{ccRCC}$ & G4 & pT3a & 7.5 & $\mathrm{Nx}$ & M1 & $\begin{array}{l}\text { Lap. Rad. Left } \\
\text { Neph. }\end{array}$ \\
\hline $\mathrm{M} / 52^{\dagger}$ & $60 \mathrm{~kg} \times 169 \mathrm{~cm}$ & Yes & 0.79 & $\mathrm{ccRCC}$ & G3 & pT1a & 3.5 & $\mathrm{Nx}$ & $\mathrm{Mx}$ & $\begin{array}{l}\text { Lap. Part. Right } \\
\text { Rob. Neph. }\end{array}$ \\
\hline $\mathrm{M} / 53^{\dagger}$ & $\mathrm{n} / \mathrm{a}$ & $\mathrm{n} / \mathrm{a}$ & 1.3 & $\mathrm{ccRCC}$ & G4 & pT3a & 11 & $\mathrm{Nx}$ & $\mathrm{Mx}$ & $\begin{array}{l}\text { Lap. Rad. Right } \\
\text { Neph. }\end{array}$ \\
\hline $\mathrm{M} / 83^{\dagger}$ & $59 \mathrm{~kg} \times 164 \mathrm{~cm}$ & Yes & 1.24 & $\mathrm{ccRCC}$ & G3 & pT3a & 7.4 & $\mathrm{Nx}$ & Mx & $\begin{array}{l}\text { Lap. Rad. Left } \\
\text { Neph. }\end{array}$ \\
\hline $\mathrm{M} / 84^{\dagger}$ & $\mathrm{n} / \mathrm{a}$ & Yes & 1.26 & ccRCC & G4 & pT2b & 10.2 & $\mathrm{Nx}$ & Mx & $\begin{array}{l}\text { Lap. Rad. Left } \\
\text { Neph. }\end{array}$ \\
\hline $\mathrm{F} / 48^{\dagger}$ & $\mathrm{n} / \mathrm{a}$ & $\mathrm{n} / \mathrm{a}$ & 1.12 & ccRCC & G3 & pT1a & 3.5 & $\mathrm{Nx}$ & $\mathrm{Mx}$ & $\begin{array}{l}\text { Lap. Part. Left } \\
\text { Neph. }\end{array}$ \\
\hline $\mathrm{M} / 45^{\dagger}$ & $\mathrm{n} / \mathrm{a}$ & $\mathrm{n} / \mathrm{a}$ & 1.16 & $\mathrm{ccRCC}$ & G2 & pT1b & 4.2 & $\mathrm{Nx}$ & $\mathrm{Mx}$ & $\begin{array}{l}\text { Lap. Part. Left } \\
\text { Rob. Neph. }\end{array}$ \\
\hline $\mathrm{M} / 72^{\dagger}$ & $\mathrm{n} / \mathrm{a}$ & $\mathrm{n} / \mathrm{a}$ & 1 & ccRCC & G3 & pT1a & 2.5 & $\mathrm{Nx}$ & $\mathrm{Mx}$ & $\begin{array}{l}\text { Lap. Part. Right } \\
\text { Rob. Neph. }\end{array}$ \\
\hline $\mathrm{M} / 73^{\dagger}$ & $\mathrm{n} / \mathrm{a}$ & $\mathrm{n} / \mathrm{a}$ & 1.08 & ccRCC & G3 & pT1a & 2.6 & $\mathrm{Nx}$ & $\mathrm{Mx}$ & $\begin{array}{l}\text { Lap. Part. Right } \\
\text { Rob. Neph. }\end{array}$ \\
\hline $\mathrm{F} / 49^{\dagger}$ & $62 \mathrm{~kg} \times 165 \mathrm{~cm}$ & Former & 1.2 & ccRCC & G2 & pT1a & 3.5 & $\mathrm{Nx}$ & $\mathrm{Mx}$ & $\begin{array}{l}\text { Lap. Rad. Right } \\
\text { Neph. }\end{array}$ \\
\hline $\mathrm{M} / 45^{\dagger}$ & $80 \mathrm{~kg} \times 175 \mathrm{~cm}$ & No & 1.26 & ccRCC & G2 & pT3a & 11 & No & M1 & $\begin{array}{c}\text { Open Rad. Right } \\
\text { Neph. }\end{array}$ \\
\hline $\mathrm{F} / 68^{\dagger}$ & $70 \mathrm{~kg} \mathrm{x} 171 \mathrm{~cm}$ & Yes & 1.7 & ccRCC & G1 & pT1b & 5.5 & $\mathrm{Nx}$ & $\mathrm{Mx}$ & $\begin{array}{l}\text { Lap. Rad. Right } \\
\text { Neph. }\end{array}$ \\
\hline
\end{tabular}

M: male; F: female; Metast: metastasis; Nx: regional lymph nodes cannot be assessed; N0: no regional lymph node metastasis; Mx: distant metastasis cannot be evaluated; M1: distant metastasis evaluated; Lap: laparoscopic;

Rad: radical; Neph: nephrectomy; Part: partial; Rob: robotical; N/a: not available; *: Belonging to the first cohort; **: Belonging to the first cohort for which also follow-up samples are available; $\uparrow$ : Belonging to the second cohort.

in urine specimens from ccRCC patients when compared with healthy donors. Of note the urine expression levels of miR-21-5p and miR-221-3p resulted unchanged in these cohorts of ccRCC patients. These results not only support the identification of miR-210-3p as non-invasive biomarker for ccRCC management, as also suggested by $\mathrm{Li}$ and colleagues, but also highlight that only specific onco-miRNAs are accumulated in urine specimens of
ccRCC patients. Of note, the urine level of miR-210-3p resulted significantly reduced during follow-up samples highlighting this onco-miRNA as potential biomarker useful not only for diagnosis but also to assess complete resection or response to treatment of ccRCC patients.

In conclusion, this study evidences a number of miRNAs, which are altered in ccRCC tissues and urine specimens, emerging as putative non-invasive 
biomarkers for ccRCC management. Further investigation including larger cohorts of patients will allow evaluating the associations existing between miRNAs levels and insurgence of metastasis, evidencing the strength of these biomarkers in the monitoring of tumor progression in ccRCC.
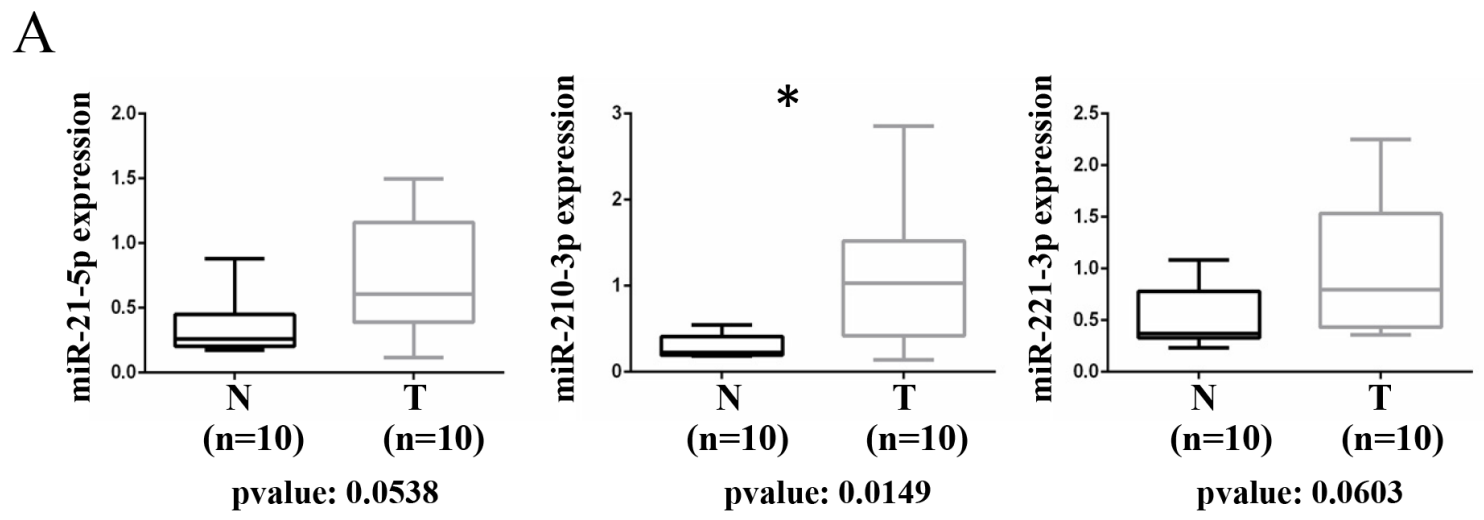

B

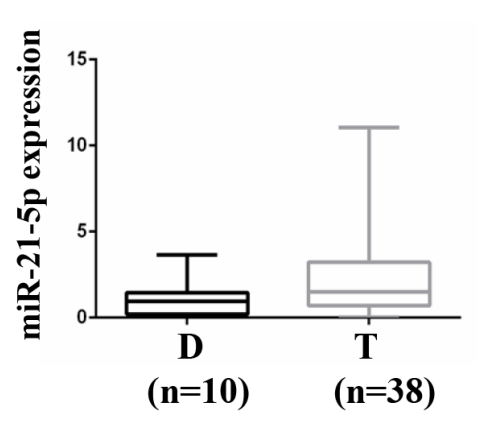

pvalue: 0.1235

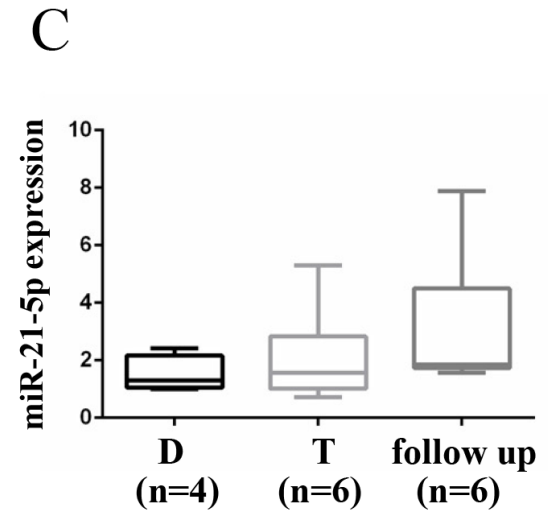

pvalue: 0.5612 pvalue: 0.0788

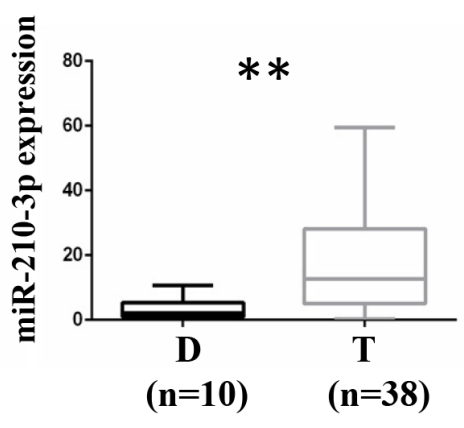

pvalue: 0.0081

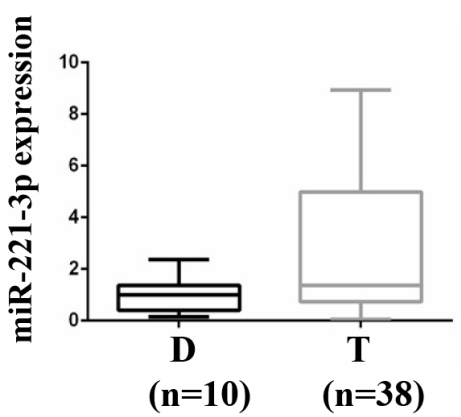

pvalue: 0.0561

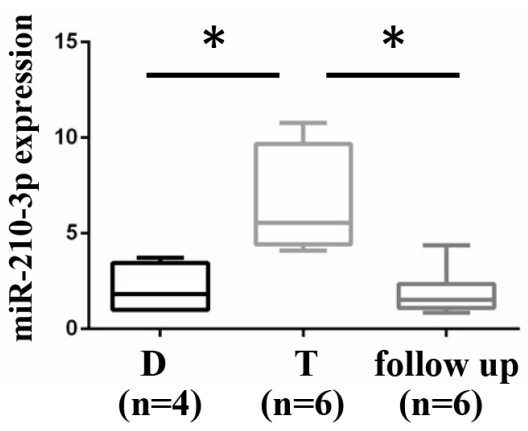

pvalue: 0.0168 pvalue: 0.0186

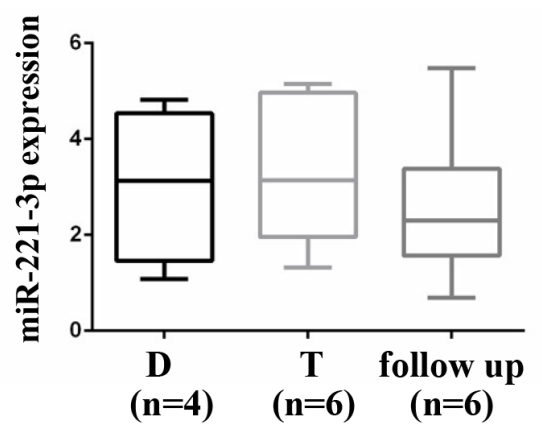

pvalue: 0.7962 pvalue: 0.3406

Figure 1: Evaluation of microRNAs levels in fresh frozen tissues and urine specimens from ccRCC patients. (A) Boxplot showing the modulation of miR-21-5p, miR-210-3p and miR-221-3p in a cohort of 10 matched fresh frozen tissues from ccRCC patients. A total of 10 matched ccRCC tumor $(\mathrm{T})$ and adjacent normal tissue $(\mathrm{N})$ samples were analyzed by RT-qPCR. The expression value of each miRNA was normalized over the average of SNORD61, SNORD68 and RNU6-2 expression through z-scores method. p-value was calculated by paired t-test and a value of $\mathrm{P} \leq 0.05(*)$ was considered statistically significant. (B) Box plots showing the modulation miR-21$5 \mathrm{p}, \mathrm{miR}-210-3 \mathrm{p}$ and miR-221-3p in urine specimens collected from $38 \mathrm{ccRCC}$ patients at the time of surgery (Tumor) and from 10 healthy donors samples (Donors) by RT-qPCR and normalized relative to the expression level of Spike-In Control through $\Delta \Delta$ CT method. p-value was calculated by unpaired t-test and a value of $\mathrm{P} \leq 0.01(* *)$ was considered statistically significant. (C) Box plot showing the expression level of miR-21-5p, miR-210-3p, miR-221-3p in urine collected from 6 patients at time of surgery (T), during follow-up (15 months as median of time from surgery) and from 4 healthy donors samples by RT-qPCR and normalized relative to the expression level of Spike-In Control through $\Delta \Delta C$ T method. $\mathrm{p}$-value was calculated by unpaired t-test and a value of $\mathrm{P} \leq 0.05\left({ }^{*}\right)$ was considered statistically significant. 


\section{MATERIALS AND METHODS}

\section{Patients and urine samples collection}

This study includes two independent cohorts of ccRCC patients (totally 38 patients) who underwent surgical resection between March 2015 and March 2017 (as described in Table 1). For the 10 ccRCC patients of the first cohort, fresh frozen-matched tumoral and normal peritumoral kidney tissues were also considered. Urine specimens were collected at the time of surgery from all the patients; for 6 of the ccRCC patients urine was collected also during follow-up (with 15 months as median of time from surgery). In particular, urine samples were frozen within 30 minutes from collection and stored at $-80^{\circ} \mathrm{C}$ until RNA extraction. Patients included in the study were not treated with any neo-adjuvant therapy before surgery. The surgery procedures performed as curative treatment for these patients were: Laparoscopic Radical Right or Left Nephrectomy in 20 cases $(52,63 \%)$, Laparoscopic Partial Right or Left Nephrectomy in 17 cases $(44,73 \%)$ and Open Radical Right Nephrectomy in only 1 case $(2,63 \%)$. Twenty-five patients were male $(65.8 \%)$ and 13 patients were female $(34.2 \%)$ with a median age of 64.5 years old (range 36-84) and a median Serum Creatinin concentration of $1.00 \mathrm{mg} / \mathrm{dl}$ (range 0.66-2.70). All the cases presented a clear cell histotype of RCC at the histological examination and according to Fuhrman's grade classification, 19 cases (50\%) were G2 grade, 13 cases $(34.21 \%)$ were $\mathrm{G} 3$ grade, 5 cases $(13.16 \%)$ were G4 grade and only 1 case $(2.63 \%)$ was G1 grade. As main risk factor, smoking habit was taken into account in 30 patients for which this information was available; among these, 12 patients $(40 \%)$ were cigarette smokers, 5 patients $(16.67 \%)$ have a history of tabagism and 13 patients $(43.33 \%)$ were non-smokers (Table 1$)$.

Urine samples were also collected from two groups of healthy donors of 4 and 6 individuals with characteristics comparable to the ccRCC patients included in the study (median age: 60.5 ; males: $60 \%$ and females: $40 \%)$.

\section{RNA extraction and microRNA expression analysis}

Fresh Frozen samples were homogenized by gentle dissociator (Miltenyi Biotec) in $700 \mu \mathrm{l}$ of Qiazol (Qiagen, Chatsworth, CA) and RNA was extracted following the manufacturer's instruction. The concentration and purity of total RNA were assessed using a Nanodrop TM 1000 spectrophotometer (Nanodrop Technologies, Wilmington, DE, USA).

A quantity of $150 \mathrm{ng}$ of total RNA was reverse transcribed in $20 \mu \mathrm{l}$ using miScript II RT kit (Qiagen,
Chatsworth) and $1 \mu \mathrm{l}$ of cDNA dilution (1:5) was used for quantitative Real time PCR (RT-qPCR) experiments.

PCR quantification analysis of the SNORD61, SNORD68, RNU6-2 and miRNAs miR-21-5p, miR210-3p, miR-221-3p, miR-185-5p and miR-145-5p, was performed using the miScript SYBR Green PCR kit (Qiagen, Chatsworth) with the miScript Primer Assay Hs-SNORD61 (\#MS00033705), SNORD68 (\#MS00033712), RNU6B-2 (\#MS00033740), Hs-miR21-5p (\#MS00009079), Hs-miR-210-3p (\#MS00003801), Hs-miR-221-3p (\#MS00003857), Hs-miR-185-5p (\#MS00003647) and Hs-miR-145-5p (\#MS00003528) (Qiagen, Chatsworth, CA, USA). All reactions were performed in triplicate. Data were analyzed by quantitative Real time PCR relative to a standard curve; standard curve was performed with serial dilution of a reference cDNA obtained from RNA extracted from a tumor sample. For the analysis $\mathrm{z}$-score were calculated for all expression value to standardize the data. Subsequently, z-score values of SNORD61, SNORD68, RNU6-2 were averaged and used to normalize the expression values of each miRNA. The $p$ value was calculated by using a parametric test with paired data.

Total RNA from $200 \mu \mathrm{l}$ of urine samples was extracted using miRNAeasy serum/plasma kit (Qiagen, Chatsworth, CA) following the manufacturer's instructions. The concentration purity and quality of total RNA were assessed using a Nanodrop TM 1000 spectrophotometer (Nanodrop Technologies, Wilmington, DE, USA). A quantity of $25 \mathrm{ng}$ of total RNA was reverse transcribed in $10 \mu \mathrm{l}$ using miScript II RT kit (Qiagen, Chatsworth) and $1 \mu \mathrm{l}$ of cDNA dilution (1:5) was used for quantitative Real time PCR (RT-qPCR) experiments. Quantification of miRNAs miR-21-5p, miR-210-3p, miR-221-3p was carried out by miScript Primer Assay (Qiagen, Chatsworth, CA), normalizing over the Spike-In Control (\#219610) (Qiagen, Chatsworth, CA) through $\triangle \Delta \mathrm{CT}$ method and using the miScript SYBR Green PCR kit (Qiagen, Chatsworth, CA). All reactions were performed in triplicate. microRNA expression was evaluated on urine samples from each of the two ccRCC cohorts and compared to that of the two groups of urine samples from healthy donors (first cohort vs. 4 donors; second cohort vs. 6 donors). Data were then collected and presented in box plots.

\section{Author contributions}

Vincenzo Petrozza, Antonio Luigi Pastore, Giovanni Palleschi, Manuela Costantini, Giuseppe Simone, Michele Gallucci and Antonio Carbone provided ccRCC samples and contributed to experimental design and writing; Claudia Tito, Natale Porta, Serena Ricci and Chiara Marigliano performed experiments; Angelina Di Carlo contributed to experimental design and writing; Francesco Fazi designed and performed experiments and wrote the paper. 


\section{CONFLICTS OF INTEREST}

No potential conflicts of interest were disclosed.

\section{FUNDING}

Contribution of Sapienza University of Rome to VP was greatly appreciated.

\section{REFERENCES}

1. Siegel R, Naishadham D, Jemal A. Cancer statistics, 2013. CA Cancer J Clin. 2013; 63: 11-30.

2. Lee CT, Katz J, Fearn PA, Russo P. Mode of presentation of renal cell carcinoma provides prognostic information. Urol Oncol. 2002; 7: 135-140.

3. Ferlay J, Autier P, Boniol M, Heanue M, Colombet M, Boyle P. Estimates of the cancer incidence and mortality in Europe in 2006. Ann Oncol. 2007; 18: 581-592.

4. MacLennan S, Imamura M, Lapitan MC, Omar MI, Lam TB, Hilvano-Cabungcal AM, Royle P, Stewart F, MacLennan G, MacLennan SJ, Canfield SE, McClinton S, Griffiths TR, et al. Systematic review of oncological outcomes following surgical management of localised renal cancer. Eur Urol. 2012; 61: 972-993.

5. Escudier B, Albiges L, Sonpavde G. Optimal management of metastatic renal cell carcinoma: current status. Drugs. 2013; 73: 427-438.

6. Hsieh JJ, Chen D, Wang PI, Marker M, Redzematovic A, Chen YB, Selcuklu SD, Weinhold N, Bouvier N, Huberman KH, Bhanot U, Chevinsky MS, Patel P, et al. Genomic biomarkers of a randomized trial comparing first-line everolimus and sunitinib in patients with metastatic renal cell carcinoma. Eur Urol. 2017; 71: 405-414.

7. Choudhury Y, Wei X, Chu YH, Ng LG, Tan HS, Koh V, Thike AA, Poon E, Ng QS, Toh CK, Kanesvaran R, Tan PH, Tan MH. A multigene assay identifying distinct prognostic subtypes of clear cell renal cell carcinoma with differential response to tyrosine kinase inhibition. Eur Urol. 2015; 67: 17-20.

8. van der Mijn JC, Mier JW, Broxterman HJ, Verheul HM. Predictive biomarkers in renal cell cancer: insights in drug resistance mechanisms. Drug Resist Updat. 2014; 17: 77-88.

9. Escudier B. Advanced renal cell carcinoma: current and emerging management strategies. Drugs. 2007; 67: 1257-1264.

10. Hayes J, Peruzzi PP, Lawler S. MicroRNAs in cancer: biomarkers, functions and therapy. Trends Mol Med. 2014; 20: 460-469.

11. Ganci F, Vico C, Korita E, Sacconi A, Gallo E, Mori F, Cambria A, Russo E, Anile M, Vitolo D, Pescarmona E, Blandino R, Facciolo F, et al. MicroRNA expression profiling of thymic epithelial tumors. Lung Cancer. 2014; 85: 197-204.

12. Osanto S, Qin Y, Buermans HP, Berkers J, Lerut E, Goeman JJ, van Poppel H. Genome-wide microRNA expression analysis of clear cell renal cell carcinoma by next generation deep sequencing. PLoS One. 2012; 7: e38298.

13. Cancer Genome Atlas Research Network. Comprehensive molecular characterization of clear cell renal cell carcinoma. Nature. 2013; 499: 43-49.

14. Christinat Y, Krek W. Integrated genomic analysis identifies subclasses and prognosis signatures of kidney cancer. Oncotarget. 2015; 6: 10521-10531. doi: 10.18632/ oncotarget.3294.

15. Ge YZ, Wu R, Xin H, Zhu M, Lu TZ, Liu H, Xu Z, Yu P, Zhao YC, Li MH, Hu ZK, Zhao Y, Zhong B, et al. A tumorspecific microRNA signature predicts survival in clear cell renal cell carcinoma. J Cancer Res Clin Oncol. 2015; 141: 1291-1299.

16. Fendler A, Stephan C, Yousef GM, Kristiansen G, Jung $\mathrm{K}$. The translational potential of microRNAs as biofluid markers of urological tumours. Nat Rev Urol. 2016; 13: 734-752.

17. Gu L, Li H, Chen L, Ma X, Gao Y, Li X, Zhang Y, Fan Y, Zhang X. MicroRNAs as prognostic molecular signatures in renal cell carcinoma: a systematic review and metaanalysis. Oncotarget. 2015; 6: 32545-32560. doi: 10.18632/ oncotarget.5324.

18. Li G, Zhao A, Peoch M, Cottier M, Mottet N. Detection of urinary cell-free miR-210 as a potential tool of liquid biopsy for clear cell renal cell carcinoma. Urol Oncol. 2017; 35: 294-299.

19. Petrozza V, Carbone A, Bellissimo T, Porta N, Palleschi G, Pastore AL, Di Carlo A, Della Rocca C, Fazi F. Oncogenic microRNAs characterization in clear cell renal cell carcinoma. Int J Mol Sci. 2015; 16: 29219-29225.

20. Pastore AL, Palleschi G, Silvestri L, Moschese D, Ricci S, Petrozza V, Carbone A, Di Carlo A. Serum and urine biomarkers for human renal cell carcinoma. Dis Markers. 2015; 2015: 251403.

21. Lou N, Ruan AM, Qiu B, Bao L, Xu YC, Zhao Y, Sun RL, Zhang ST, Xu GH, Ruan HL, Yuan CF, Han WW, Shi HC, et al. miR-144-3p as a novel plasma diagnostic biomarker for clear cell renal cell carcinoma. Urol Oncol. 2017; 35 : 36 e $37-36$ e 14.

22. Urquidi V, Netherton M, Gomes-Giacoia E, Serie DJ, Eckel-Passow J, Rosser CJ, Goodison S. A microRNA biomarker panel for the non-invasive detection of bladder cancer. Oncotarget. 2016; 7: 86290-86299. doi: 10.18632/ oncotarget.13382.

23. Zhang X, Zhang Y, Liu X, Fang A, Wang J, Yang Y, Wang L, Du L, Wang C. Direct quantitative detection for cell-free miR-155 in urine: a potential role in diagnosis and prognosis 
for non-muscle invasive bladder cancer. Oncotarget. 2016; 7: 3255-3266. doi: 10.18632/oncotarget.6487.

24. Yoshino H, Yonemori M, Miyamoto K, Tatarano S, Kofuji S, Nohata N, Nakagawa M, Enokida H. microRNA-210-3p depletion by CRISPR/Cas9 promoted tumorigenesis through revival of TWIST1 in renal cell carcinoma. Oncotarget. 2017; 8: 20881-20894. doi: 10.18632/oncotarget.14930.

25. Mlcochova H, Hezova R, Stanik M, Slaby O. Urine microRNAs as potential noninvasive biomarkers in urologic cancers. Urol Oncol. 2014; 32: 41 e41-49. 